\title{
Primordial Black Holes from Collapsing Antimatter
}

\author{
Gábor Etesi ${ }^{1}$ (D) \\ Accepted: 20 December 2021 / Published online: 5 February 2022 \\ (c) The Author(s) 2022
}

\begin{abstract}
In this paper a simple (i.e. free of fine-tuning, etc.) new mechanism for primordial black hole formation based on the collapse of large antimatter systems in the early Universe is introduced. A peculiarity of this process is that, compared to their material counterparts, the collapse of large antimatter systems takes much less time due to the reversed thermodynamics of antimatter, an idea which has been proposed in our earlier paper Etesi (2021). This model has several testable predictions. The first is that the photon-baryon ratio is roughly computable and is equal to $3.03 \times 10^{9}$ which is quite close to its experimentally confirmed value. The second is that the mass of black holes arising from this mechanism is at least $10^{5}-10^{6} M_{\odot}$ hence they contribute to the super- or hypermassive end of the primordial black hole mass spectrum. The third prediction is that these sort of primordial black holes constitute at least $20 \%$ of dark matter. Last but not least the observed current asymmetry of matter and antimatter, even if their presence in the Universe was symmetric in the beginning, acquires a natural explanation, too.
\end{abstract}

Keywords Primordial black holes $\cdot$ Matter-antimatter asymmetry $\cdot$ Second law

\section{Introduction}

Phenomena of the physical world, as immediately given to us, appear in inexhaustable structures and formations of matter. At first sight a simple quantitative comprehension is achieved by understanding how much amount of matter a given fixed spatial region can accommodate. Approaching this way despite the endless possibilities one discovers two limits for matter formation: the lower universal limit is realized by an elementary particle (more precisely a relativistic quantum field) while the upper one is attained by a black hole; then one quickly arrives at the standard traditional and apparently disconnected territories of relativistic quantum field theory and the theory of gravity (general relativity). However this straightforward division into a linear and monotonic scheme extending from the "smallest" (which is something like an atomic thing) towards the "largest" (which is something like a very different celestial thing) is too narrow. While the masses and sizes of

Gábor Etesi

etesi@math.bme.hu

1 Department of Geometry, Mathematical Institute, Faculty of Science, Budapest University of Technology and Economics, Egry J. u. 1, H ép., H-1111 Budapest, Hungary 
elementary particles are indeed very small and are sharply restricted by yet unknown quantization rules such that the formers are below $m_{\text {Planck }} \approx 10^{-5} \mathrm{~g}$ and the latters are above the corresponding Compton wave length $r_{\text {Planck }} \approx 10^{-35} \mathrm{~m}$, on the contrary black holes can in principle bear an arbitrary mass and size ranging from $m_{\text {Planck }}$ with corresponding Schwarzschild radius $r_{\text {Planck }}$ up to $1.2 \times 10^{43} \mathrm{~g}$ and $1.8 \times 10^{13} \mathrm{~m}$ (the data of the recently directly observed supermassive central black hole in the M87 giant elliptic galaxy) or even higher. Heavy black holes, whose existence has already been experimentally verified, indeed resemble astrophysical objects and have suitable origin however smaller-and-smaller black holes, if exist, exhibit more-and-more particlelike features; therefore the hypothetical borderline entity with mass $m_{\text {Planck }}$ and size $r_{\text {Planck }}$ can equally well be treated as either an extremely heavy particle or an extremely light black hole. Thus the apparently linear hierarchy of matter organization in Nature with its two limits rather would take a circular shape (if e.g. small black holes indeed exist).

A promising, even experimentaly confirmed candidate for a reservoir of small(er) black holes is cosmic dark matter. It is very likely a dark cocktail of various currently only hypothetical physical entities such as primordial black holes (PBHs) including evaporation remnants and yet mainly unknown weakly interacting massive particles (WIMPs) like neutrinos, axions, etc. The idea of a primordial black hole was introduced by Hawking 50 years ago Hawking (1971b) and it was recognized soon (Carr and Hawking, 1974, p. 403) that the majority of matter might exist in the form of (primordial) black holes in the present Universe. There has been an intense debate recently among cosmologists and particle physicists concerning the ratio of the various dark matter candidates (it is impossible to give a complete list of references here therefore we refer here and from now on at other places to the excellent up-to-date review Carr and Kühnel (2020) and the hundreds of references therein). Although we are still far from being conclusive according to diverse and accurate observations at least four mass windows are open for a primordial black hole abundance: these are the $10^{-16}-10^{-10} M_{\odot}$ together with the $10^{-6}-10^{-5} M_{\odot}$ windows in the small black hole range, the $10-10^{3} M_{\odot}$ window in the medium range and the larger than $10^{13} M_{\odot}$ spectrum in the hypermassive range, cf. (Carr and Kühnel, 2020, Figure 1). It is not unreasonable that even our outer Solar System harbours a small black hole Scholtz and Unwin (2020).

Sudden and violent primordial black hole formations during the course of the evolution of the Universe are usually associated with phase transitions of all kinds, cf. Carr and Kühnel (2020) and in particular Asaka et al. (2004); Carr et al. (2019); García-Bellido et al. (2019). The general pattern is that the later the black hole formation occurs the higher the achieved black hole mass is Carr and Hawking (1974). In this paper a particular late-time phase transition, namely the photon recombination time around 380.000 years after the Big Bang is examined from the point of view of massive primordial black hole formation. Our aim here is to offer a new mechanism based on a reversed thermodynamical behaviour of antimatter introduced in our earlier paper Etesi (2021).

Already in 1939 von Weizsäcker noted that the obvious but subjective difference between the past and future in our temporal experiences gains an objective substantiation by understanding the very content of the second law of thermodymanics von Weizsäcker (1939). This understanding, among other consequences, would make the artificial division of time i.e. duration into a collection of disjoint and durationless instants, as motivated by the usual set-theoretic model of the continuum and assumed everywhere in physics, doubtful. While contemplating along these lines about the structure of time and its role played in current physical theories (for a survey cf. e.g. Kuzemsky (2020); Sachs (1987)), the idea that macroscopic antimatter follows a reversed form of the second law of thermodynamics 
has been proposed Etesi (2021). Our suggestion is perhaps not independent of Feynman's original ideas around 1947 that antiparticles should be regarded as ordinary (i.e. positive energy) particles but travelling backwards in time Feynman (1949). The Proposal (see its discussion in Sect. 2 below) implies that even if the states of a macroscopic matter and a macroscopic antimatter system are strictly identical on a "snapshot" taken at a fixed but purely hypothetically existing moment, their observable temporal behaviour is yet different and this difference is characterized by the usual and the reversed form of the second law. If one is indeed willing to accept that identical mechanical states might imply different temporal behaviour for matter and antimatter in their thermodynamical limit then one in fact questions a basic concept of Hamiltonian mechanics, namely the state. However the original Hamiltonian notion of a state which works well in traditional (i.e. e.g. antimatter-free) mechanics became already problematic in the 1930-40's (as Feynman's idea also indicates) when physicists tried again to work out a model for the classical or the relativistic quantum field theoretic electron which is free of self-energy and other divergence problems. ${ }^{1}$ We do not intend to discuss here the deep problems arising from the division of the continuum into disjoint constituents Baez (2016); rather point out that even if the Proposal sounds weird it cannot be easily refuted by assuming its validity and then seeking a contradiction with some part of classical mechanics: for the Proposal is the logical negation of the usual second law finding such a contradiction would be logically equivalent to a proof of the second law of thermodynamics from the laws of mechanics which is a very difficult (if not impossible) problem since Boltzmann's times. In our opinion the validity of the Proposal is an experimental question.

Hopefully motivated with these introductory remarks in some extent, in this paper, in the realm of the structure of time we shall revisit the problem of the absence of antimatter from the Universe on macroscopic scales. What we are going to do is simple: instead of trying to derive the second law of thermodynamics from other abstract laws of theoretical physics we shall regard it as an underivable, irreducible, fundamental law expressing a basic empirical evidence about the temporal behaviour of macroscopic matter. The consequent application of considering the second law as an empirical evidence imposes at least one non-trivial constraint on its appearance in the physical world namely in its known form it is immediately applicable only to ordinary matter for this is the only form of matter which we have direct phenomenological contact with. Then we exhibit one plausible argument, based on various principles of theoretical physics but referring to the aforementioned observational validity of the second law, that the second law continues to hold for large antimatter systems but in a reversed form. Their converse thermodynamic behaviour could then lead to their swift confinement behind black hole event horizons hence to the absence of antimatter on marcroscopic scales from the Universe (also cf. Boyle et al., 2018; Cohen and Kaplan, 1987; Toussaint et al., 1979). Consequently the problem of missing antimatter Cohen et al. (1998), Steigman (1976) naturally connects with the formation and frequency of black holes in the early Universe Asaka et al. (2004), Carr et al. (2019), García-Bellido et al. (2019). The mechanism we offer here sounds appealing for it does not require any fine-tuning or new asymmetric mechanism around Big Bang times to explain the macroscopic matter-antimatter asymmetry, as usually assumed in string theoretic and other approaches.

\footnotetext{
1 As an aside we remark that the difficulty of assigning intermediate (i.e. non-asymptotic) states to interacting relativistic quantum fields was one of the theoretical — among other, including experimental—reasons why quantum field theory became a theory of scattering instead of the theory of states like traditional quantum mechanics Blum (2017).
} 
The paper is organized as follows. In Sect. 2 for completeness and the reader's convenience we recall from Etesi (2021) the Proposal but in a substantially improved form. Then in Sect. 3 we apply it for the early Universe and introduce a new primordial black hole formation mechanism.

\section{A Proposal and its Consequence}

The idea of an elementary antiparticle had quite unexpectedly dropped out from the theoretical efforts to reconcile the basic principles of special relativity and quantum mechanics; shortly thereafter their individual existence was verified by cosmic ray detectors, nuclear reactors and high energy particle colliders. However no physical experiment or even any kind of human experience in the broadest sense exists so far which could provide some phenomenological insight into the macroscopic i.e., thermodynamical properties of pure antimatter built up from bound states of these antiparticles. Even assuming that the basic principles of (classical or quantum) statistical mechanics continue to hold for physical systems consisting of pure antimatter-and confessing that the derivation of the second law of thermodynamics from these principles is problematic yet - the thermodynamical behaviour of such alien macroscopic physical systems is, honestly speaking, unknown to us presently. Therefore we are not in contradiction with any element of our contemporary description of physical reality if we make the following bit counterintuitive

Proposal Let $\mathscr{S}_{\text {antimatter }}$ be a closed physical system consisting of pure antimatter (in the low energy and thermodynamical limit). Then the entropy $S$ of this system never increases in time i.e., $\Delta S\left(\mathscr{S}_{\text {antimatter }}\right) \leqq 0$.

In our opinion the ultimate validity or invalidity of the Proposal is an experimental question; it can be surely decided by experiments designed to unfold the dynamics of large antimatter systems.

The property of being (anti)matter is Lorentz invariant i.e., it cannot be switched by Lorentz transformations. Therefore, as an immediate consistency check we note that the Proposal is Lorentz invariant as well. This means that for any physical system $\mathscr{S}$ (evolving forward in time) the sign of its entropy change, i.e. $\operatorname{sign}(\Delta S(\mathscr{S})= \pm 1$ or 0 in case of equilibrium, is invariant under Lorentz transformations despite that the entropy function $S(\mathscr{S})$ itself as usually defined in phenomenological thermodynamics or statistical mechanics is not obviously a Lorentz scalar. Indeed, let $\mathscr{S}$ be a macroscopic physical system evolving along a future-directed non-spacelike congruence in Minkowski space-time and let $\gamma$ be a (co-moving or nearby, etc.) observer i.e. a future-directed timelike curve; define the entropy change of $\mathscr{S}$ with respect to $\gamma$ as the difference of the entropy of $\mathscr{S}$ at a system-event observed as the later event $\gamma(\tau+\varepsilon)$ minus the entropy of $\mathscr{S}$ at a system-event observed as the earlier event $\gamma(\tau)$ i.e.,

$$
\Delta S(\mathscr{S}, \gamma):=S(\mathscr{S}, \gamma(\tau+\varepsilon))-S(\mathscr{S}, \gamma(\tau))
$$

Let $\gamma^{\prime}$ be another (perhaps distant) observer and define $\Delta S\left(\mathscr{S}, \gamma^{\prime}\right)$ analogously. Since $\mathscr{S}$ evolves causally its two system events above are not spacelike separated consequently the observer $\gamma^{\prime}$ records them in the same causal order: it observes the system-event corresponding to $\gamma(\tau+\varepsilon)$ later than the system-event corresponding to $\gamma(\tau)$, too. Consequently even if perhaps $\Delta S\left(\mathscr{S}, \gamma^{\prime}\right) \neq \Delta S(\mathscr{S}, \gamma)$, we are sure that at least $\operatorname{sign}\left(\Delta S\left(\mathscr{S}, \gamma^{\prime}\right)\right)=\operatorname{sign}(\Delta S(\mathscr{S}, \gamma))$ i.e. $\operatorname{sign}(\Delta S(\mathscr{S}))$ is well-defined as stated. 
Regarding its current experimental status, although as a hint for the Proposal it is worth revisiting the already observed Kabir (1999) asymmetry between the processes $K^{0} \rightarrow \bar{K}^{0}$ and $\bar{K}^{0} \rightarrow K^{0}$, one has to acknowledge that we are still very far from a sharp experimental evaluation of the Proposal. This is because despite the discovery of antimatter more than a half century ago only a very few types of antielements (namely ${ }^{1} \overline{\mathrm{H}},{ }^{2} \overline{\mathrm{H}},{ }^{3} \overline{\mathrm{H}}$ and ${ }^{3} \overline{\mathrm{He}},{ }^{4} \overline{\mathrm{He}}$ ) could have been produced so far and typically for very short times and in atomistic amounts only. However after taking an overview of these efforts we can select for our purposes the most relevant one namely the ALPHA experiment at CERN, which is a very exciting ongoing experiment exhibiting lot of new (but on theoretical grounds expected) facts about antihydrogen atoms, to see whether or not the communicated results can be used to support or reject the Proposal. Latest results have been reported in Baker et al. (2021) however from our point of view, i.e. regarding some technical background details, we shall revisit an older paper ALPHA Collaboration (2011) from 2011 too. Recall that the original as well as present aim of the ALPHA together with the AEgIS experiment at CERN is to create and trap antihydrogen isotopes in order to carefully compare their physical properties with their ordinary counterparts. These physical properties are their lifetime (i.e. stability), spectrum, moreover soon gravitational characteristics like their mass and gravitational acceleration, too. In other words, and one should keep in mind this, the ALPHA and AEgIS experiments first of all have been technically designed to obtain precise information about properties of individual antiatoms. Nevertheless, since during the experiments thermal ensemble of antihydrogen atoms have regularly been produced, one expects to gain at least a marginal insight into their collective behaviour, too.

The ALPHA experiment roughly goes as follows (ALPHA Collaboration, 2011, Figure 1) and (Baker et al., 2021, Figure 1). Using CERN's antiproton and positron accelerators and decelerators, soft antiproton and positron beams are injected into a tube of $280 \mathrm{~mm}$ axial length and of $44.35 \mathrm{~mm}$ diameter. The interior of this tube is vacuous and kept at low temperature, has optical access, and fulfilled with a strong magnetic field; it is actually a magnetic trap which is capable to confine those antihydrogen atoms which, after the recombination of the antiproton-positron plasma, can sufficiently rapidly cool down via advanced auxiliary laser cooling. Impressively, these atoms then can be trapped for several hours inside the vacuum tube to perform experiments. The vacuum tube is surrounded by silicon detectors to record final annihilations caused by interactions with the environment. This environment contains the tube's boundary, residual gases inside the vacuum tube as well as incoming particles from cosmic radiation and other accidental sources. Thus of course this environment consists of ordinary matter having standard thermodynamical properties. What from our viewpoint relevant is the values of the following three parameters in every individual attempt or run of the experiment: the number $N$ of trapped antihydrogen atoms, their temperature $T$ and their confinement time $t$. Although the cumulative value of $N$ was reported to be about 1000 in ALPHA Collaboration (2011); Baker et al. (2021), its average value in individual attempts (i.e. the situation when antiatoms are under sharp observational control), as summarized in (ALPHA Collaboration, 2011, Table 1 and Figure 2) (but not available in Baker et al., 2021), was $N \approx 1$. Regarding the further parameters $T \approx 10$ $-100 \mathrm{mK}$ and $t \approx$ several hours. We can now make three observations. Firstly, despite the vacuum tube's macroscopical volume $V \approx 3.95 \times 10^{-5} \mathrm{~m}^{3}$ the entropy $S=S(N, T, V)$ of this antihydrogen gas system is practically zero (in accord with the third law of thermodynamics); consequently $S$ is practically constant despite the long observational time $t$ of any attempt. Secondly, the antihydrogen gas as observed in the ALPHA experiment cannot be considered as an ideal gas since its individual antihydrogen atoms are magnetically trapped generating strong correlations between their e.g. speed components (see e.g. (ALPHA 
Collaboration, 2011, Figure 3)). Therefore the truely free random motion of antihydrogen atoms in space, which is essential to study the temporal behaviour of their population's entropy, by design is not guaranteed even during long observational times. Thirdly and perhaps most importantly: the detectation of the position of an antihydrogen atom is based on its annihilation with the ordinary matter environment hence the position measurement procedure itself makes a strong ordinary thermodynamical influence on the antimatter system. (From our point of interest a more favourable position measurement protocoll should use e.g. low frequency i.e. soft photon scattering on the antihydrogen gas). To summarize: as, in our opinion, nobody could confirm the validity of the second law for normal hydrogen in a situation analogous to the ALPHA experiment, the current stage of this experiment is not suitable to challenge the Proposal on an objective basis, too.

Regarding its current theoretical status, proving or disproving the Proposal using the apparatus of theoretical physics and mathematics is at least as difficult as proving or disproving the ordinary second law. This is because the Proposal is precisely the logical negation of the ordinary second law of thermodynamics; consequently a falsification of the Proposal by assuming its validity and then arriving at a contradiction with some part of theoretical physics is logically equivalent to a proof of the second law (that is deriving it from the laws of classical or quantum statistical mechanics) by contradiction.

Having seen that challenging the Proposal experimentally or theoretically is not straightforward, we would rather like to offer here one heuristic argument for its validity. In the following derivation of the Proposal the validity of the second law as an empirical evidence about macroscopic ordinary matter systems will play a crucial role. This explains the absence of any kind of microscopic calculations from the considerations below: from the circle of our arguments it follows that the converse thermodynamical properties of antimatter is recognizable only macroscopically i.e. compared to that of ordinary particles, we are not going to modify the microscopic dynamics of antiparticles at all! Putting differently, one can say that the converse second law for antimatter is non-derivable from timesymmetric microscopic physics in exactly the same way as the ordinary second law is not derivable from it (yet).

An argument based on the CPT theorem of relativistic quantum field theories. In light of our accurate experimental evidences, we have no reason to doubt the validity of the basic rules of relativistic quantum field theory when applied to both matter and antimatter. One of the most fundamental results of the relativistic quantum field theoretic description of physical reality is the $C P T$ theorem which states that the triple action of charge conjugation $C$, spatial reflection $P$ and time direction reversal $T$, when applied to a relativistic particle system, realizes a symmetry of it (cf. e.g. [53, Chapter I.5.8]). Since macroscopic matter is built up from the bound states of these relativistic particles it is reasonable to expect that the $C P T$ theorem continues to hold for low energy macroscopic physical systems in an appropriate effective form (for strongly related considerations cf. Klimenko and Maas (2014)). We will assume two things: firstly that the physical system is built up from atomic (or molecular) matter in the low energy thermodynamical limit (this is certainly not true at the elementary particle level). This implies that the proposed $C P T$ violating mechanisms mainly based on various field oscillations between flavour-eigenstates (cf. e.g. Benatti and Floreanini, 2000; Capolupo et al., 2019; Gago et al., 2001; Guzzo et al., 2019; Lisi et al., 2000; Simonov et al., 2019) are negligable in a good approximation: these $C P T$ violating effects are proportional to the mass difference between the flavour eigenstates of these free elementary particle fields but the occurence or the interaction with atomic matter of these states is negligable in the low energy limit. Secondly we assume that the parity transformation $P$ alone is already a symmetry of a physical system in the low energy thermodynamical 
limit (this is also not true at the elementary particle level). This assumption implies that performing $P$ on an existing low energy macroscopic physical system we obtain an existing low energy macroscopic physical system.

Consider now an ordinary closed physical system $\mathscr{S}_{\text {matter }}$ consisting of pure (normal) matter in the low energy thermodynamical limit, evolving forward in time. Therefore, as a theoretical consequence, the $C P T$ theorem in its effective form is applicable to $\mathscr{S}_{\text {matter }}$ and tells us that

$$
\operatorname{CPT}\left(\mathscr{S}_{\text {matter }}\right)=\mathscr{S}_{\text {matter }} .
$$

Another empirical evidence about $\mathscr{S}_{\text {matter }}$ is the validity of the second law of thermodynamics:

$$
\Delta S\left(\mathscr{S}_{\text {matter }}\right) \geqq 0
$$

i.e., the entropy of a closed physical system consisting of pure ordinary matter in the low energy thermodynamical limit never decreases. Putting together these we get

$$
\Delta S\left(C P T\left(\mathscr{S}_{\text {matter }}\right)\right) \geqq 0 .
$$

However, accepting the validity of the $C P T$ theorem in the low energy thermodynamical limit in an effective form discussed above, the $C P T$ transformation converts a closed physical system of matter evolving forward in time into a closed physical system containing (spatially reflected, hence existing) antimatter evolving backward in time i.e.,

$$
C P T\left(\mathscr{S}_{\text {matter }}\right)=\mathscr{S}_{\text {antimatter in reversed time }} \text {. }
$$

Therefore the last inequality implies

$$
\Delta S\left(\mathscr{S}_{\text {antimatter in reversed time }}\right) \geqq 0
$$

i.e., the entropy of an antimatter system never decreases in reversed time hence switching back to ordinary time we come up with

$$
\Delta S\left(\mathscr{S}_{\text {antimatter }}\right) \leqq 0
$$

leading to the Proposal.

A comment on $C P T$ violation: recently there has been a debate concerning the (in)validity of the $C P T$ theorem on cosmological scales, in the presence of weak interaction, strong gravitational fields, etc., etc. (cf. e.g. Benatti and Floreanini (2000); Boyle et al. (2018); Capolupo et al. (2019); Gago et al. (2001); Guzzo et al. (2019); Kabir (1999); Lisi et al. (2000); Simonov et al. (2019)). Since our previous naive derivation of the Proposal refers to the $C P T$ theorem and our considerations ahead deal with black holes in the early Universe it is worth addressing this issue here for a moment.

Let $X, Y$ be some elementary particle states, denote by $P_{X Y}(t)$ the probability of the occurence of the forward-in-time-process $X \rightarrow Y$ at a laboratory time $t$ and likewise $P_{Y X}(t)$ the converse but also forward-in-time-process $Y \rightarrow X$. Introduce Kabir (1999) the timeasymmetry parameter

$$
A_{T}(t):=\frac{P_{X Y}(t)-P_{Y X}(t)}{P_{X Y}(t)+P_{Y X}(t)}
$$


If for example $X=K^{0}$ and $Y=\bar{K}^{0}$ are the neutral kaon and its antiparticle states then the observed violation of $C P$ in the kaon system together with the theoretical assumption of the validity of $C P T$ explains the observed $T$ violation i.e. $A_{T} \neq 0$ in kaon experiments Kabir (1999). However this temporal asymmetry already can be used alone to argue in favour to the Proposal namely that the forward-in-time dynamics of kaon systems differs from that of their antiparticle counterparts (and this temporal asymmetry is generated by the weak interaction). Accordingly, most of the proposed $C P T$ violating mechanisms derive the violation itself from the theoretical assumption that in certain situations (e.g. large free particle systems in the presence of gravity Simonov et al. (2019)) $C P$ holds true but finding theoretically that in these situations $T$ fails because $A_{T} \neq 0$. Therefore one may wonder whether or not in these situations the temporal asymmetry alone can be used directly (i.e. without referring to the $C P T$ theorem as we did before) to argue for (some form of) the Proposal.

To close this section we discuss one consequence which plays a crucial role in our considerations ahead. We begin with clarifying that from now on by "accepting the Proposal" in case of a macroscopic antimatter system $\mathscr{S}_{\text {antimatter }}$ we shall mean the following: this system obeys the same physical laws describing its physical states as its corresponding ordinary matter system $\mathscr{S}_{\text {matter }}$ defined by $\mathscr{S}_{\text {matter }}:=C\left(\mathscr{S}_{\text {antimatter }}\right)$ where $C$ is the charge conjugation operator; however the physical laws describing the dynamics of $\mathscr{S}_{\text {antimatter }}$ might be different and are characterized by the Proposal in an appropriate way. Then let us consider a closed macroscopical system $\mathscr{S}_{\text {antimatter }}$ built up from pure antimatter only hence not disturbed by recombination, etc. effects; thus the time evolution of $\mathscr{S}_{\text {antimatter }}$ is governed only by its own gravitational, electromagnetic and thermodynamical phenomena. Accepting the Proposal therefore $\mathscr{S}_{\text {antimatter }}$ obeys the same equation of state (expressing a phenomenological relation between its energy, temperature, pressure, volume, etc.) as its corresponding macroscopic ordinary matter system $\mathscr{S}_{\text {matter }}$ however, unlike this latter, $\mathscr{S}_{\text {antimatter }}$ tends to evolve into more-and-more ordered states in time by its own dynamics. Since in case of $\mathscr{S}_{\text {matter }}$ the evolution into more-and-more disordered states often includes spatial expansion, the evolution of $\mathscr{S}_{\text {antimatter }}$ into more-and-more ordered states could imply its stronger tendency for spatial contraction. Consequently, in sharp contrast to an ordinary matter system, the structural tendency of $\mathscr{S}_{\text {antimatter }}$ for spatial contraction in its own gravitational field could be enhanced by the functional tendency of $\mathscr{S}_{\text {antimatter }}$ for spatial contraction thanks to its reversed thermodynamics. ${ }^{2}$

After these rather abstract general arguments let us examine the Proposal and its consequences from a physically more realistic direction.

\footnotetext{
${ }^{2}$ It is illustrive to regard the structural and functional characters as sort of spatial and temporal projections, respectively, of a common abstract "character" of a physical system. In this language we can say that physical systems possess an abstract "contraction tendency" whose structural and functional manifestations are the gravity and the thermodynamical phenomena, respectively (cf. Verlinde's idea of entropic gravity Verlinde (2011)) and they attenuate each other in the case of ordinary matter systems while enhance each other in the case of antimatter systems.
} 


\section{An Application to Primordial Black Hole Formation}

Consider the early Universe about the time when the last relevant, namely the electronpositron, spontaneous pair creation process stops because of cooling. Recall that in the radiation epoch $T \sim t^{-1 / 2}$ and for definiteness and simplicity we assume the temperature is about $T_{0} \approx 10^{9} \mathrm{~K}$ and the time is about $t_{0} \approx 10 \mathrm{~s}$ after the Big Bang. Note that the physical description of the Universe already falls fully within the classical and non-(special)relativistic realm at these late times. Assuming the most natural initial conditions namely that the Universe was created with perfect particle-antiparticle symmetry and with precisely zero total electric charge we suppose that the Universe consists of an equal amount of baryonic-leptonic matter and antibaryonic-antileptonic antimatter of vanishing total electric charge surrounded with electromagnetic radiation, all in thermal equilibrium. Let us therefore model the whole situation with a closed classical thermodynamical system $\mathscr{S}$ consisting of a finite spatial region fulfilled with a matter-photon-antimatter plasma in thermal equilibrium (whether or not $\mathscr{S}$ can indeed be assumed to be closed will be addressed shortly). The expansion of the Universe is adiabatic hence its entropy is unchanged during (at least short times of the) expansion. We summarize all of these by writing symbolically $\mathscr{S}=\mathscr{S}_{\text {matter+radiation+antimatter }}$ satisfying

$$
\Delta S\left(\mathscr{S}_{\text {matter + radiation + antimatter }}\right)=0 \text { around } t_{0} \approx 10 \mathrm{~s} .
$$

We assume that the spatial region has volume $V_{0}$ which is much larger than the Debye length in the fulfilling plasma i.e. $V_{0} \gg \lambda_{D}^{3} \sim\left(\frac{T_{0}}{n_{0}}\right)^{3 / 2}$ where $n_{0}$ is the number density of the most dilute charged particle constituent in $V_{0}$ consequently the long-range electromagnetic interactions in the system are negligable because of screening-off. In a good approximation the only interaction between the matter-photon-antimatter subcomponents is annihilation or recombination of the various particle-antiparticle pairs. Since by our initial assumptions the electric charge of the observable Universe is zero at large temporal and spatial scales, as a single indicator for these various pair recombination processes we are going to monitor the main electron-positron recombination process, namely $e^{-} e^{+} \rightarrow 2 \gamma$ only. However we note that this restriction, especially if neutrino effects are to be considered as well, can easily be relaxed in the following considerations if necessary.

Let us explore the time evolution of the system satisfying (1). Denoting by $t \gtrsim t_{0}$ the time variable let $V(t)$ be the volume of $\mathscr{S}_{\text {matter+radiation+antimatter }}$ and $N(t)$ the number of electrons (or positrons) in it at a moment. More precisely let $N(t)$ be the expectation value at $t$ of the number of electrons in $V(t)$. Actually the true number of electrons in $V(t)$ essentially never coincides with the abstract number $N(t)$ rather takes its value somewhere in the interval $[N(t)-\sqrt{N(t)}, N(t)+\sqrt{N(t)}]$ due to thermal fluctuations i.e. accidentally entering and exiting particles. In other words strictly speaking our system is not closed. However if $N(t)$ is not the actual value but only the expectation value of the particle number, as we demand, then its very property is that it is independent of thermal fluctuations. Consequently with this definition of $N(t)$ the system $\mathscr{S}_{\text {matter+radiation+antimatter can indeed be }}$ assumed to be closed. These obvious but important remarks also imply that $N(t)$ depends on $t$ only through particle reactions which in our simple model means the single $e^{-} e^{+} \rightarrow 2 \gamma$ process alone.

Next let us therefore derive the evolution equation for this process. Of course a necessary condition for an electron-positron pair to annihilate in a fixed instant is that they should approach each other well in space; we capture this quantitatively by 
saying that if $\sigma(t)$ denotes the cross-section of the $e^{-} e^{+} \rightarrow 2 \gamma$ process then one particle must approximately stay within a ball of radius $\sqrt{\sigma(t)}$ about its antiparticle or vice versa during a short time interval $\Delta t$; consequently if $v(t)=\left|\mathbf{v}_{e^{ \pm}}(t)\right|$ is the average speed of a particle and $\Delta t<\sqrt{\sigma(t)} / v(t)$ then the effective annihilation volume is not $V(t)$ but $v(t) \Delta t \sigma(t) N(t)$ only. Assuming uniform distribution the number of particles in this volume is $(v(t) \Delta t \sigma(t) N(t) / V(t)) N(t)$ which is therefore in a good approximation is equal to the number $N(t)-N(t+\Delta t)=-\Delta N(t)$ of annihilating pairs during $\Delta t$. Consequently letting $\Delta t \rightarrow 0$ the electron (or positron) number decreases according to

$$
\frac{\mathrm{d} N(t)}{\mathrm{d} t}=-\frac{v(t) \sigma(t)}{V(t)} N^{2}(t)
$$

The calculation of the cross-section of the $e^{-} e^{+} \rightarrow 2 \gamma$ process in the plane wave approximation (i.e. when the long range Coulomb forces are neglected) is a classical result of Dirac; since the plasma is already non-relativistic his quite complicated formula (Heitler 1954, Equation (7) in Chapter V, §27) reduces to its simple non-relativistic limit

$$
\sigma(t) \approx \pi r_{0}^{2} \frac{c}{2 w(t)}
$$

where $r_{0}=e^{2} / m_{e} c^{2} \approx 2.82 \times 10^{-15} \mathrm{~m}$ is the classical electron radius and $w(t)$ is the average speed of the colliding particles in their center-of-mass system hence

$$
w(t)=\frac{1}{4 \pi} \int_{S^{2}}\left|\mathbf{v}_{e^{ \pm}}(t)-\frac{\mathbf{v}_{e^{-}}(t)+\mathbf{v}_{e^{+}}(t)}{2}\right| \mathrm{d} \Omega=\frac{1}{4 \pi} \int_{S^{2}} \frac{\left|\mathbf{v}_{e^{-}}(t)-\mathbf{v}_{e^{+}}(t)\right|}{2} \mathrm{~d} \Omega=\frac{2}{3} v(t) .
$$

Note that the cross-section increases with time.

Consider first the radiation epoch $10 \mathrm{~s} \lesssim t \lesssim 70.000$ a (here "a" stands for "years" as usual). Then $R(t) \sim \sqrt{t}$ implying $V(t) \sim t^{3 / 2}$. Therefore

$$
\left\{\begin{array}{l}
\dot{N}(t)=-\frac{3 \pi r_{0}^{2} t_{0}^{3 / 2}}{4 V_{0}} t^{-3 / 2} N^{2}(t) \\
N\left(t_{0}\right)=N_{0}
\end{array}\right.
$$

where $V(t)=V_{0} \cdot\left(t / t_{0}\right)^{3 / 2}$ with $V_{0}>0$ being the initial volume at $t_{0} \approx 10 \mathrm{~s}$. Moreover $N_{0}>0$ is the initial particle number. The particular solution hence looks like

$$
N(t)=\left(\frac{1}{N_{0}}+\frac{3 \pi r_{0}^{2} c t_{0}^{3 / 2}}{2 V_{0}}\left(\frac{1}{\sqrt{t_{0}}}-\frac{1}{\sqrt{t}}\right)\right)^{-1}
$$

satisfying

$$
\lim _{t \rightarrow+\infty} N(t)=\left(\frac{1}{N_{0}}+\frac{3 \pi r_{0}^{2} c t_{0}}{2 V_{0}}\right)^{-1}
$$

consequently having, quite surprisingly, a non-vanishing asymptotics thanks to the expansion.

Next, in the matter epoch i.e. when 70.000 a $t \lesssim 1.38 \times 10^{10}$ a, then $R(t) \sim t^{2 / 3}$ yields $V(t) \sim t^{2}$. Therefore in the matter epoch 


$$
\left\{\begin{array}{l}
\dot{N}(t)=-\frac{3 \pi r_{0}^{2} c t_{1}^{2}}{4 V_{1}} t^{-2} N^{2}(t) \\
N\left(t_{1}\right)=N_{1}
\end{array}\right.
$$

where $t_{1} \approx 70.000$ a and $V_{1}=V_{0} \cdot\left(t_{1} / t_{0}\right)^{3 / 2}>0$ but now $V(t)=V_{1} \cdot\left(t / t_{1}\right)^{2}$. Moreover $N_{1}$ is the electron number at $t_{1}$. Note that by (2) surely $N_{1}>0$ hence the corresponding matching particular solution in the matter epoch again looks like

$$
N(t)=\left(\frac{1}{N_{1}}+\frac{3 \pi r_{0}^{2} c t_{1}^{2}}{4 V_{1}}\left(\frac{1}{t_{1}}-\frac{1}{t}\right)\right)^{-1}
$$

yielding

$$
\lim _{t \rightarrow+\infty} N(t)=\left(\frac{1}{N_{1}}+\frac{3 \pi r_{0}^{2} c t_{1}}{4 V_{1}}\right)^{-1}
$$

hence has finite asymptotics, too. Note that without expansion i.e. putting $V(t)=$ const. both solutions above would have trivial asymptotics $N(t) \sim t^{-1}$ i.e. the annihilation would be complete in this case.

Taking into account the electron-positron number asymptotics (2) and (3) together with the fact that the Universe is electrically neutral on large temporal and spatial scales, hence qualitatively all other particle (except probably the various neutrino) densities must follow moreor-less the same asymptotics, we end up with a rather surprising possibility: despite that their annihilation cross-section increases with passing time, in the sufficiently rapidly expanding Universe the matter and antimatter constituents do not annihilate completely. Although the previous considerations have been straightforward, the idea itself that antimatter could survive the early violent history of the Universe might look strange at first sight (although we note that various non-trivial freeze-out scenarios have already been studied by other authors, too cf. e.g. Murphy et al. (2005), Thomas et al. (2020)). Fortunately testable predictions derivable from this model help to measure the validity of this possibility. Perhaps the most directly accessable as well as measurable consequence is the photon-baryon ratio which is supposed to be somewhere between $10^{9}$ and $10^{10}$ in light of astronomical observations and cosmological considerations.

Thus let us make a digression here and see how this ratio looks like in our model. The initial number of electrons is $N_{0}$ at the early moment $t_{0} \approx 10 \mathrm{~s}$ meanwhile its late time limit is (3) and their difference had been annihilated mainly into photons. Thus the quantity $1 / \eta_{0}:=\left(N_{0}-\lim _{t \rightarrow+\infty} N(t)\right) / \lim _{t \rightarrow+\infty} N(t)$ measures the magnitude of the number of recombined electron-positron pairs hence the photon-electron ratio. In its calculation just for simplicity the late time limit (2) juxtaposed with (3) can be replaced with the latter one alone if we write $t_{0}, N_{0}, V_{0}$ instead of $t_{1}, N_{1}, V_{1}$ in (3). In this way we find

$$
\frac{1}{\eta_{0}}=\frac{N_{0}}{\lim _{t \rightarrow+\infty} N(t)}-1=1+\frac{3 \pi r_{0}^{2} c t_{0} N_{0}}{4 V_{0}}-1=\frac{3 \pi r_{0}^{2} c t_{0} \rho_{0}}{13 k_{B} T_{0}}
$$

where in the last step we inserted $\varepsilon_{0}=(3 / 2) k_{B} T_{0}$ and wrote $\rho_{0}=(13 / 6) \varepsilon_{0} N_{0} / V_{0}$ for the baryonic matter energy density of the early Universe taking into account that the total $e^{-}+p^{+}+n^{0}$ number in the initial volume $V_{0}$ was about $M_{0} \approx(1+1+1 / 6) N_{0}=(13 / 6) N_{0}$ at $t_{0} \approx 10 \mathrm{~s}$. Concerning the numerical value of $\rho_{0}$ we assume that the current total energy 
density of the Universe is $5.2 \mathrm{GeV} / \mathrm{m}^{3}$ having about $4 \%$ baryonic matter constituent hence $\rho_{0} \approx 0.21\left(T_{0} / 2.71 \mathrm{~K}\right)^{3} \mathrm{GeV} / \mathrm{m}^{3}$ with $T_{0} \approx 10^{9} \mathrm{~K}$. Plugging all the constants into the formula we obtain $1 / \eta_{0} \approx 2.12 \times 10^{9}$. From this number the photon-baryon ratio arises as follows. The total $e^{-}+p^{+}+n^{0}$ number at late times is $M \approx(1+1+1 / 7) \lim _{t \rightarrow+\infty} N(t)$ thus the total number of annihilating pairs is $\left(M_{0}-M\right) / M \approx 1 / \eta_{0}$. Now let us do photon counting. The $e^{-} e^{+} \rightarrow 2 \gamma$ process produces 2 photons from every annihilating pair. In addition to this we have to consider the leading low energy nucleon-antinucleon annihilations as well which are all the $p^{+} p^{-}, p^{+} \bar{n}^{0}, n^{0} p^{-}$and $n^{0} \bar{n}^{0} \rightarrow X$ processes. Referring to accurate particle collider results Backenstoss (1983) the average number of emitted photons in any of these pair recombinations is $\approx 3.93$. Abandoning other decay channels (but for a broader survey of $N \bar{N}$ annihilations cf. Klempt et al. (2005)) these nucleon processes together with $e^{-} e^{+} \rightarrow 2 \gamma$ produce in average $\left(3.93+2 \times 3.93 / 7+3.93 / 7^{2}+2\right) / 5 \approx 1.43$ photons. Consequently the photon-baryon ratio in our model looks like

$$
\frac{1}{\eta} \approx \frac{1.43}{\eta_{0}} \approx 3.03 \times 10^{9}
$$

which, taking into account the very rough estimates and simplifications we made throughout, ${ }^{3}$ is close to its latest experimentally confirmed value $1 / \eta \approx 1.67 \times 10^{9}$, cf. Ade et al. (2014); Steigman (2007).

Having seen that the recombination of matter with antimatter in the early Universe, such that the latter did not fully disappear from the stage during the course of this recombination era, produces a convincing photon-baryon ratio, one is unavoidably forced to say something on the following well-known fundamental problem formulated by Sakharov 60 years ago Sakharov $(1967,1991)$ : if this was indeed the story then what happened to antimatter Shaposhnikov (2000)? Why large antimatter "islands" are not observable Cohen et al. (1998); Steigman (1976) in the current Universe in spite of the obvious evidence that similar ordinary matter clouds do exist? This is the point where we evoke the Proposal, which has not been used so far, i.e. apply it for large but isolated antimatter domains in the early Universe whose existence at least in the past follows from our previous considerations.

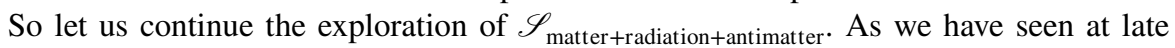
times it yet contains both matter and antimatter which essentially do not interact; consequently the original system splits into closed (or almost closed) subsystems what we write symbolically as

$$
\mathscr{S}_{\text {matter+radiation+antimatter }}=\mathscr{S}_{\text {matter }}+\mathscr{S}_{\text {radiation }}+\mathscr{S}_{\text {antimatter }} .
$$

Hence by the (sub)additivity of the entropy the equilibrium equation (1) decouples as well consequently at for instance the hydrogen recombination time we re-write it as

$$
\Delta S\left(\mathscr{S}_{\text {matter }}\right)+\Delta S\left(\mathscr{S}_{\text {radiation }}\right)+\Delta S\left(\mathscr{S}_{\text {antimatter }}\right)=0 \text { around } t \approx 380.000 \mathrm{a} .
$$

Recall that in the matter epoch $T \sim t^{-2 / 3}$ more precisely at this moment $T \approx 3000 \mathrm{~K}$ and the system contains neutral components (mainly photon gas and atomic hydrogen, helium

\footnotetext{
3 Surely the most important of these simplifications was the systematic suppression of all neutrino effects including the decays $e^{-} e^{+} \rightarrow v_{x} \bar{v}_{x}$ where $x=e, \mu, \tau$. However as a consistency check note that since $\rho_{0} \sim T_{0}^{3}$ and $T_{0} \sim t_{0}^{-1 / 2}$ in the radiation era, at least the number $\eta_{0}=13 k_{B} T_{0} /\left(3 \pi r_{0}^{2} c t_{0} \rho_{0}\right)$ hence the baryon-photon ratio $\eta$ itself is independent of the particular choice $1 \mathrm{~s} \lesssim t_{0} \lesssim 10 \mathrm{~s}$ for the initial value of time and the corresponding temperature $10^{10} \mathrm{~K} \gtrsim T_{0} \gtrsim 10^{9} \mathrm{~K}$ in the leptonic epoch we began with.
} 
together with their antimatter counterparts) only hence $\mathscr{S}_{\text {matter }}+\mathscr{S}_{\text {radiation }}+\mathscr{S}_{\text {antimatter }}$ can be treated well with the traditional tools of ideal gas theory in phenomenological thermodynamics from now on. The thermal equilibrium of the global system however does not necessarily implies the thermal equilibrium of its (weakly interacting) subsystems. This means that we have to examine them separately. Regarding $\mathscr{S}_{\text {matter }}$ an empirical evidence (i.e. not a theoretical deduction) about this and only this subsystem is again the validity of the second law

$$
\Delta S\left(\mathscr{S}_{\text {matter }}\right) \geqq 0
$$

Within our closed system the next subsystem is $\mathscr{S}_{\text {radiation }}$ consisting of pure thermal radiation in equilibrium at temperature $T\left(\mathscr{S}_{\text {radiation }}\right)$ occupying a volume $V\left(\mathscr{S}_{\text {radiation }}\right) \lesssim V(t)$. By the Stefan-Boltzmann law $S\left(\mathscr{S}_{\text {radiation }}\right)=\frac{4}{3} a T^{3}\left(\mathscr{S}_{\text {radiation }}\right) V\left(\mathscr{S}_{\text {radiation }}\right)$. But $T\left(\mathscr{S}_{\text {radiation }}\right) \sim t^{-1 / 2}$ and $V\left(\mathscr{S}_{\text {radiation }}\right) \sim t^{3 / 2}$ in the radiation era while $T\left(\mathscr{S}_{\text {radiation }}\right) \sim t^{-2 / 3}$ and $V\left(\mathscr{S}_{\text {radiation }}\right) \sim t^{2}$ in the matter era. Thus we find that

$$
\Delta S\left(\mathscr{S}_{\text {radiation }}\right)=0
$$

which is of course in agreement with observations, too. (Note that this equality can be obtained via the Proposal as well by applying it together with the usual second law on a closed physical system consisting of particles equal to their own antiparticles like $\mathscr{S}_{\text {radiation }}$; then indeed we can write both $\mathscr{S}_{\text {radiation }}=\mathscr{S}_{\text {antimatter }}$ and $\mathscr{S}_{\text {radiation }}=\mathscr{S}_{\text {matter }}$ implying both $\Delta S\left(\mathscr{S}_{\text {radiation }}\right)=\Delta S\left(\mathscr{S}_{\text {antimatter }}\right) \leqq 0$ and $\Delta S\left(\mathscr{S}_{\text {radiation }}\right)=\Delta S\left(\mathscr{S}_{\text {matter }}\right) \geqq 0$ hence (6). $)$

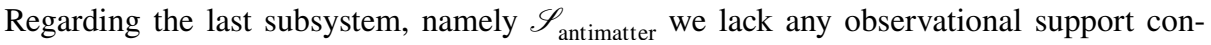
cerning its temporal behaviour however comparing (5) and (6) with (4) we can conclude that

$$
\Delta S\left(\mathscr{S}_{\text {antimatter }}\right) \leqq 0
$$

in accord with the Proposal in the particular case of large isolated antimatter systems in the early but already low energy Universe.

Geometrically the system $\mathscr{S}_{\text {matter }}+\mathscr{S}_{\text {radiation }}+\mathscr{S}_{\text {antimatter }}$ on a long range of macroscopic scales contains both matter and antimatter subregions of more-or-less equal volumes surrounded by electromagnetic radiation. To be more visual and taking into account the overall gravitational contraction as well, we can assume that within the finite volume $V(t)$ at a fixed time the spatial subregion occupied by $\mathscr{S}_{\text {matter }}$ is a (disjoint) union of finitely many 3 dimensional balls of different radii, mainly proportional to the Jeans length under these conditions Battaner (1996), and likewise for $\mathscr{S}_{\text {antimatter }}$ such that the complementum of these balls is fulfilled with $\mathscr{S}_{\text {radiation. }}$. Having set up this natural picture let us consider the further time evolution of the system when $t \gtrsim 380.000$ a. The temporal evolutions of $\mathscr{S}_{\text {matter }}$ and $\mathscr{S}_{\text {antimatter }}$, which have been parallel up to this point, sharply split from now on. This is in some sense not surprising because $t \approx 380.000$ a is a crucial phase transition, namely the photon recombination, time in the history of the Universe.

Regarding $\mathscr{S}_{\text {matter }}$ its individual ball constituents undergo standard star formation by gravitational contraction and further fragmentation. Recall that the first stage of this complex evolution is always an isothermal process in which a given ball radiates heat to its environment $\mathscr{S}_{\text {radiation }}$ during contraction. Consequently these balls are not closed systems 
taking into account this interaction. ${ }^{4}$ Nevertheless being of course an ordinary gas ball in a thermal bath observable, as an empirical evidence the second law surely applies to the inthis-way-interacting system $\mathscr{S}_{\text {matter }}+\mathscr{S}_{\text {radiation }}$ hence

$$
\Delta S\left(\mathscr{S}_{\text {matter }}+\mathscr{S}_{\text {radiation }}\right) \geqq 0 \text {. }
$$

Moreover neither the volume change nor the companying thermal radiation of a gas ball in $\mathscr{S}_{\text {matter }}$ has effect on the volume or the temperature of its vast environment described by $\mathscr{S}_{\text {radiation }}$ hence in a good approximation $\Delta S\left(\mathscr{S}_{\text {matter }}+\mathscr{S}_{\text {radiation }}\right) \approx \Delta S\left(\mathscr{S}_{\text {matter }}\right)+\Delta S\left(\mathscr{S}_{\text {radiation }}\right)$ thus via (5) and (6) we find that in fact $\Delta S\left(\mathscr{S}_{\text {matter }}\right) \geqq 0$. That is, despite the radiative interaction, we can assume that $\mathscr{S}_{\text {matter }}$ alone satisfies the second law as usually assumed in standard textbooks on star formation Zeldovich (1971).

After these preliminary observations take any particular ball within $\mathscr{S}_{\text {matter }}$ and treat it as a massive gas ball having volume $V$, particle number $N$, mass $m$, total energy $E$ and gravitational potential energy $U$ (hence kinetic energy $K=E-U$ ). Its entropy looks in the standard way like

$$
S(V, E)=N k_{B}\left(\log \frac{V}{N}+\frac{3}{2} \log \frac{E-U}{N}+\text { const. }\right) .
$$

On substituting $V=(4 \pi / 3) R^{3}$ and the Newtonian potential energy $U=-G m^{2} / R$ we can re-write it as

$$
\begin{aligned}
S(R, E) & =N k_{B}\left(3 \log \frac{R}{N^{1 / 3}}+\frac{3}{2} \log \frac{1}{N}\left(E+\frac{G m^{2}}{R}\right)+\text { const. }\right) \\
& =\frac{3}{2} N k_{B}\left(\log \frac{E R^{2}+G m^{2} R}{N^{5 / 3}}+\text { const. }\right)
\end{aligned}
$$

showing that $0<R<+\infty$ if $E \geqq 0$ or $0<R<-G m^{2} / E$ if $E<0$. It turns out that the shape of the entropy function depends crucially on these two cases. Indeed, by solving the equation

$$
\left.\frac{\partial S(R, E)}{\partial R}\right|_{E=\text { const. }}=\frac{3}{2} N k_{B} \frac{2 E R+G m^{2}}{E R^{2}+G m^{2} R}=0
$$

it readily follows that if $E \geqq 0$ i.e. the system is gravitationally not bounded then the entropy is a monotonly increasing function of $R$ hence without local extrema. Therefore taking any $0<R_{\text {initial }}<+\infty$ and applying the second law for $\mathscr{S}_{\text {matter }}$ we find that $R \rightarrow+\infty$ hence the system is unstable in the usual sense: in order to maximize its entropy, a ball performs an unbounded spatial expansion as one would expect. The second possibility is that $E<0$ i.e. the system is gravitationally bounded then there is precisely one maximum of the entropy function at

$$
R=-\frac{G m^{2}}{2 E}
$$

\footnotetext{
${ }^{4}$ But of course this radiative interaction is different from the one based on annihilation considered before but found to be already irrelevant at this asymptotic stage.
} 
Therefore, depending on $R_{\text {initial }}$ the system performs a finite expansion, remains unchanged or performs a finite contraction (by absorbing or releasing thermal radiation such that its temperature remains constant) in order to reach $R=-G m^{2} / 2 E$ where $\mathscr{S}_{\text {matter }}$ attains its maximal entropy hence stable equilibrium state. This is the well-known isothermal phase of ordinary star formation.

Now let us see how this analysis works for antimatter gas balls. Accepting the Proposal $^{5} \mathscr{S}_{\text {antimatter }}$, which system in our astrophysical situation therefore describes a similar massive and in the beginning cold pure antimatter ideal gas arranged into balls of various radii, satisfies the usual equation of state $p V=N k_{B} T$. Consequently taking one of its ball constituents the previous calculations work here as well. However this time we find a different temporal behaviour. The first possibility is when $E \geqq 0$ hence this ball is gravitationally unbounded; then taking into account the reversed second law (7) we find that starting with any $R_{\text {initial }}$ the radius of the ball behaves like $R \rightarrow 0$ that is, the antimatter gas ball is unstable again however in a reversed way: unlike an ordinary matter ball it performs an unbounded contraction. The second possibility is that the ball is gravitationally bounded i.e. $E<0$ and is already small i.e. $0<R_{\text {initial }}<-G m^{2} / 2 E$. Applying (7) we find again that $R \rightarrow 0$ i.e. a sufficiently small bounded antimatter gas ball is unstable in the sense that it undergoes an unbounded contraction, too. The third possibility is that the ball is gravitationally bounded i.e. $E<0$ and its volume is fine-tuned i.e. precisely $R_{\text {initial }}=-G m^{2} / 2 E$. Then this is an equilibrium of having maximal entropy hence is unstable in light of (7). The fourth possibility is that the ball is gravitationally bounded i.e. $E<0$ but is not small i.e. $-G m^{2} / 2 E<R_{\text {initial }}<-G m^{2} / E$. Then by (7) it is unstable again but, quite surprisingly $R \rightarrow-G m^{2} / E$ i.e. it would undergo a finite expansion such that the ball reaches a finite size. However the finite mass (and total energy) antimatter ball would have unbounded entropy in this limiting state which is a contradiction (if we regard the entropy as the logarithm of the number of microstates of a finite system). Hence the last two possibilities are ruled out which simply means that in our model the radius $R$, mass $m$ and total energy $E<0$ of a gravitationally bounded antimatter gas ball, when taking into account its dynamics i.e. temporal behaviour as well, always satisfies the inequality $R<-G m^{2} / 2 E$. As a consistency check we remark that this maximal size is the half of the allowed maximal size for a gravitationally bounded ordinary matter ball of the same mass and negative total energy; this factor is reasonable in light of the stronger contraction tendency of antimatter: the idea we have stressed throughout the paper. Nevertheless, and to summarize, we have seen that in our model all of these gravitationally unbounded or bounded antimatter balls suffer from a contractive instability.

The characteristic collapsing time of this contractive instability can be estimated by the aid of the Proposal alone (i.e. neglecting all other physical mechanisms for simplicity) if the interaction with the environment is also taken into account. Suppose $E_{\text {initial }}<0$ and $0<R_{\text {initial }}<-G m^{2} / 2 E_{\text {intial }}$ moreover that $\dot{E}<0$ hence $E<0$ throughout i.e. the gravitationally bounded ball radiates energy to its environment $\mathscr{S}_{\text {radiation }}$ during the contraction (cf. (Klimenko and Maas, 2014, pp. 1197-1198)) hence remains gravitationally bounded. Then putting $v:=\dot{R}$ applying (7) in the form

$$
\frac{\partial S}{\partial R} v+\frac{\partial S}{\partial E} \dot{E}=\dot{S} \leqq 0
$$

\footnotetext{
${ }^{5}$ Cf. the end of Section 2 what do we precisely understand by this.
} 
and taking into account that $v<0$ we find that $|v| \geqq R^{2} \dot{E} /\left(2 E R+G m^{2}\right)>0$. If we further assume that $\dot{E}=$ const. then the estimated time required to contract from $R_{\text {initial }}$ down to some $0<R<R_{\text {initial }}$ is

$$
t_{R}=\int_{0}^{t_{R}} \mathrm{~d} t=\int_{R}^{R_{\text {initial }}} \frac{\mathrm{d} r}{|v|} \leqq \int_{R}^{R_{\text {initial }}} \frac{2 E r+G m^{2}}{r^{2} \dot{E}} \mathrm{~d} r \leqq \frac{G m^{2}}{\mid \text { const. } \mid} \int_{R}^{R_{\text {initial }}} \frac{\mathrm{d} r}{r^{2}}=\frac{G m^{2}}{\mid \text { const. } \mid}\left(\frac{1}{R}-\frac{1}{R_{\text {initial }}}\right)
$$

which is finite even for the Schwarzschild radius $R=2 \mathrm{Gm} / \mathrm{c}^{2}$ of the antihydrogen ball.

Therefore taking into account their contractive instability discussed above one would expect that sufficiently massive antimatter gas clouds, compared to ordinary ones, are more capable to form black holes or enter already existing ones hence effectively feed them in very short times during the course of their dynamical evolution. Consequently, in light of the various uniqueness ("no-hair") theorems of black hole physics (cf. e.g. Heusler (1996)) pure macroscopic antimatter systems could disappear behind primordial black hole event horizons tracelessly faster in time than their ordinary counterparts. The details of why this antimaterial collapsing or feeding mechanism could be so effective are admittedly unclear at this stage of the art; perhaps the proposed reversed thermodynamics of antimatter somehow could prevent the system from friction hence the formation of high temperature radiating accretion discs, jets, etc. which are well-known refraining phenomena in case of observed compact objects swallowing normal matter. Although these important questions are open, for clarity we remark that the process itself is not in contradiction with Hawking's area theorem (cf. e.g. Bardeen et al. 1973; Hawking 1971a) because the fall of antimatter into a black hole, whatever weird its dynamical behaviour is, continues to transport further mass, electric charge and angular momentum into the black hole hence continues to increase the area of its instantaneous event horizon. ${ }^{6}$

These qualitative considerations permit to make some testable predictions for primordial black hole physics. The first is that these black holes, due to their quite late born around the recombination time $t \gtrsim 380.000 \mathrm{a}$, are expected to be very massive. The mass of these black holes are related with the typical value of $R_{\text {initial }}$, the radius of the collapsing ball examined above. Since the origin of these balls is the primordial fragmentation of the homogeneous but gravitationally unstable antihydrogen (and antihelium) gas, their radii are expected to satisfy $R_{\text {initial }} \sim \lambda_{J}$ where $\lambda_{J}$ is the Jeans length around the recombination time. Hence the expected typical initial mass is proportional to the corresponding Jeans mass $m_{J}$ which by considerations still applicable here ${ }^{7}$ is estimated in Battaner (1996) to be $m_{J} \sim 10^{5}-10^{6} M_{\odot}$. This large value is consistent with the general pattern that late-time born primordial black holes are expected to be heavier than the early-time ones. Consequently these black holes improve the less-understood super- or hypermassive end of the primordial black hole mass spectrum Carr and Kühnel (2020). Note that, on the contrary to their rapid formation, the typical high mass of these primordial black holes prevents them from too early evaporation by Hawking radiation Hawking (1975), Wald

\footnotetext{
${ }_{6}$ For example the horizon area $A=4 \pi\left(2 m^{2}-q^{2}+2 m \sqrt{m^{2}-a^{2}-q^{2}}\right)$ of the Kerr-Newman black hole is invariant under $q \leftrightarrow-q$ i.e. the action of the charge conjugation operator $C$. Consequently from the point of view of black hole mechanics as summarized in Bardeen et al. (1973) it is inessential what sort of infalling thing, i.e. matter or antimatter, feeds the black hole. This is of course in agreement with the no-hair theorems.

7 Note that the standard computation of the Jeans length and mass rests only on mechanical i.e. reversible considerations.
} 
(1975): since $t_{\text {evaporation }} \approx 2.1 \times 10^{67}\left(m_{J} / M_{\odot}\right)^{3}$ years which in this case is about $10^{82}-10^{85}$ years, these black holes do not reveal their content within our current cosmological times; consequently the details of their fate (i.e. the possible existence of evaporation remnants and their perhaps antimaterial nature, etc.) do not affect our considerations. The second prediction is that since the amount of matter is equal to antimatter and the latter had completely disappeared this way moreover the observed dark matter-baryonic matter ratio is about $5: 1$ it follows that at least approximately $20 \%$ of the dark matter exists in the form of massive primordial black holes in our model. There has been recently an intense debate on the mass spectrum and the ratio of the primordial black hole constituent of dark matter; our predictions are consistent with current observational constraints as summarized in Carr and Kühnel (2020): regarding the mass spectrum observations permit the existence of an abundance of primordial black holes in the very heavy end of the mass spectrum while regarding the ratio all possibilities are apparently open (hence our $20 \%$ looks like a good compromise between the extremes).

\section{Conclusion}

In this paper two apparently independent problems of current cosmology: the basic problem of matter-antimatter asymmetry in the present Universe and late time primordial black hole formation has been connected by a proposed reversed thermodynamical behaviour of antimatter. Within this framework the observed baryon-photon ratio has been reproduced $\left(\eta \approx 3.31 \times 10^{-10}\right.$ is our prediction) whose accuracy is convincing $\left(\eta \approx 6.05 \times 10^{-10}\right.$ is the experimental value) taking into account the simplicity and naturality of its derivation carried out here. Moreover two testable predictions concerning the average black hole masses (which is larger than $10^{5}-10^{6} M_{\odot}$ ) and the primordial black hole ratio in dark matter (which is at least $20 \%$ ) in this model has been exhibited. However the model's most appealing feature is surely a natural, effortless (i.e. free of any fine-tuning, etc.) explanation of the problem of missing antimatter.

To close we emphasize once more that the idea proposed here requires further elaboration and we also admit that all of these rough qualitative considerations might be invalidated by exploring the highly complex details of time evolution of realistic physical systems including the effect of gravity on the CPT theorem Simonov et al. (2019); however these certainly very difficult analyses are beyond the limits of this short note. Nevertheless our considerations, perhaps together with other suggested mechanisms (far from being complete cf. e.g. Arnold and McLerran, 1987; Asaka et al., 2004; Boyle et al., 2018; Buchmuller and Plumacher, 1999; Carr et al., 2019; Cohen and Kaplan, 1987; Farrar and Shaposhnikov., 1993; García-Bellido et al., 2019, 1999; Joyce et al., 1995; Matsumura et al., 2004; Toussaint et al., 1979; Weinberg, 1979), might shed a light onto the origin of the observed matter-antimatter asymmetry in the current Universe, even if matter and antimatter was produced in symmetric amounts in the Big Bang. This asymmetric mechanism together with the symmetric recombination effects could be responsible for the deficit of antimatter as well as for the rapid early galaxy formation around supermassive primordial black hole cores in the observable Universe.

Acknowledgements The author is grateful to K. Bozsonyi, K. Furgason, L. Krasznahorkai and F. Siklér for the stimulating discussions. There are no conflicts of interest to declare that are relevant to the content of this article. All the not-referenced contents in this work are fully the author's own contribution. No funds, grants, or other financial supports were received. The work meets all ethical standards applicable here. 
Funding Open access funding provided by Budapest University of Technology and Economics.

Open Access This article is licensed under a Creative Commons Attribution 4.0 International License, which permits use, sharing, adaptation, distribution and reproduction in any medium or format, as long as you give appropriate credit to the original author(s) and the source, provide a link to the Creative Commons licence, and indicate if changes were made. The images or other third party material in this article are included in the article's Creative Commons licence, unless indicated otherwise in a credit line to the material. If material is not included in the article's Creative Commons licence and your intended use is not permitted by statutory regulation or exceeds the permitted use, you will need to obtain permission directly from the copyright holder. To view a copy of this licence, visit http://creativecommons.org/licenses/by/4.0/.

\section{References}

Ade, P.A.R. et al. (2014) Planck 2013 results. XVI. Cosmological parameters, Astronomy and Astrophysics, 571, A16.

ALPHA Collaboration (2011) Confinement of antihydrogen for 1000 seconds, Nature Physics, 7, 558-564.

Arnold, P. B., \& McLerran, L. D. (1987). Sphalerons, small fluctuations and baryon number violation in electroweak theory. Physical Review D, 36, 581.

Asaka, T., Grigoriev, D., Kuzmin, V., \& Shaposhnikov, M. E. (2004). Late reheating, hadronic jets and baryogenesis. Physical Review Letter, 92, 101303.

Backenstoss, G., et al. (1983). Proton-antiproton annihilations at rest into $\pi^{0} \omega, \pi^{0} \eta, \pi^{0} \gamma, \pi^{0} \pi^{0}$ and $\pi^{0} \eta^{\prime}$. Nucl. Phys. B, 228, 424-438.

Baez, J. (2016). Struggles with the continuum, preprint, 42 pp., arXiv: 1609.0142 [math-ph] .

Baker, C. J., et al. (2021). Laser cooling of antihydrogen atoms. Nature, 592, 35-42.

Bardeen, J. M., Carter, B., \& Hawking, S. W. (1973). The four laws of black hole mechanics. Communications in Mathematical Physics, 31, 161-170.

Battaner, E. (1996). Astrophysical fluid dynamics. Cambridge: Cambridge University Press.

Benatti, F., \& Floreanini, R. (2000). Open system approach to neutrino oscillations. Journal High Energy Physical, 02, 032.

Blum, A.S. (2017). The state is not abolished, it withers away: how quantum field theory became a theory of scattering, Studies in the History and Philosophy of Modern Physics 60, 46-80 arXiv:2011.05908 [physics.hist-ph];

Boyle, L., Finn, K., \& Turok, N. (2018). CPT-symmetric universe. Physical Review Letter, 121, 251301.

Buchmuller, W., \& Plumacher, M. (1999). Matter antimatter asymmetry and neutrino properties. Physical Reports, 320, 329-339.

Capolupo, A., Giampaolo, S. M., \& Lambiase, G. (2019). Decoherence in neutrino oscillations, neutrino nature and CPT violation. Physical Letter B, 792, 298-303.

Carr, B., \& Hawking, S. W. (1974). Black holes in the early universe. Monthly Notices of the Royal Astronomical Society 168, 399-416.

Carr, B., Clesse, S., \& García-Bellido J (2019). Primordial black holes, hot-spot electroweak baryogenesis at the quark-hadron epoch, preprint, 39 pp., arXiv: 1904.02129 [astro-ph.CO] .

Carr, B., \& Kühnel, F. (2020). Primordial black holes as dark matter: recent developments. Annual Review of Nuclear and Particle Science, 70, 355-394.

Cohen, A. G., \& Kaplan, D. B. (1987). Thermodynamic generation of the baryon asymmetry. Physical Letter B, 199, 251-258.

Cohen, A. G., De Rújula, A., \& Glashow, S. L. (1998). A matter-antimatter universe? Astrophys Journal 495, 539-549.

Etesi, G. (2021). Antimatter and the second law of thermodynamics. Foundations of Science, 26, 217-224.

Farrar, G.R., \& Shaposhnikov, M.E. (1993). Baryon asymmetry of the universe in the minimal Standard Model, Physical Review Letter, 70, 2833-2836; Erratum: Physical Review Letter 71, 210.

Feynman, R. P. (1949). The theory of positrons. Physical Review, 76, 749-759.

Gago, A.M., Santos, E.M., Teves, W.J.C., \& Zukanovich Funchal, R. (2001). Quantum dissipative effects and neutrinos: current constraints and future perspectives, Physical Review, D63, 073001.

García-Bellido J., Carr, B., \& Clesse, S. (2019). A common origin for baryons and dark matter, preprint, 6 pp., arXiv: 1904.11482 [astro-ph.CO].

García-Bellido, J., Grigoriev, D., Kusenko, A., \& Shaposhnikov, M. E. (1999). Non-equilibrium electroweak baryogenesis from preheating after inflation. Physical Review D, 60, 123504. 
Guzzo, M. M., de Holanda, P. C., \& Oliveira, R. L. N. (2019). Quantum dissipation in a neutrino system propagating in vacuum and in matter. Nuclear Physical B, 908, 408-422.

Hawking, S. W. (1971). Gravitational radiation from colliding black holes. Physical Review Letter, 26, $1344-1346$.

Hawking, S. W. (1971). Gravitationally collapsed objects of very low mass. Monthly Notices of the Royal Astronomical Society 152, 75-78.

Hawking, S. W. (1975). Particle creation by black holes. Communications in Mathematical Physics 43, 199-220.

Heitler, W. (1954). The quantum theory of radiation. Oxford: Clarendon Press.

Heusler, M. (1996). Black hole uniqueness theorems, Cambridge Lecture Notes Phys. 6, Cambridge Univ. Press, Cambridge.

Joyce, M., Prokopec, T., \& Turok, N. (1995). Electroweak baryogenesis from a classical force, Physical Review Letter, 75, 1695-1698, Erratum: Physical Review Letter 75, 3375 (1995);

Kabir, P. K. (1999). Observed asymmetry in $\bar{p} p \rightarrow \pi^{+} K^{-} K^{0} / \pi^{-} K^{+} \bar{K}^{0}$ and relation to reciprocity. Physical Letter B, 459, 335-340.

Klempt, E., Batty, C., \& Richard, J.-M. (2005). The antinucleon-nucleon interaction at low energy: annihilation dynamics. Physical Reports, 413, 197-317.

Klimenko, A. Y., \& Maas, U. (2014). One antimatter-two possible thermodynamics. Entropy, 16, $1191-1210$.

Kuzemsky, A. L. (2020). In search of time lost: asymmetry of time and irreversibility in natural processes. Foundations of Science, 25, 597-645.

Lisi, E., Marrone, A., \& Montanino, D. (2000). Probing possible decoherence effects in atmospheric neutrino oscillations. Physical Review Letter 85, 1166-1169.

Matsumura, S., Dolgov, A. D., Nagataki, S., \& Sato, K. (2004). Affleck-Dine baryogenesis and heavy elements production from inhomogeneous Big Bang nucleosynthesis. Progress of Theoretical and Experimental Physics 112, 971-981.

Murphy, R. J., Share, G. H., Skibo, J. G., \& Kozlowsky, B. (2005). The physics of positron annihilation in the solar atmosphere. Astrophysical Journal Supplement Series 161, 495-519.

Sachs, R. G. (1987). The physics of time-reversal. Chicago: University Chicago Press.

Sakharov, A.D. (1967). Violation of CP invariance, C asymmetry, and baryon asymmetry of the universe, Pisma Zh. Eksp. Teor. Fiz. 5, 32-35, [English trans.: Usp. Fiz. Nauk. 161 61-64 (1991)].

Sakharov, A. D. (1991). Baryon asymmetry of the universe. Soviet Physics Uspekhi 34, 417-421.

Scholtz, J., \& Unwin, J. (2020). What if planet 9 is a primordial black hole? Physical Review Letter 125, 051103.

Shaposhnikov, M. E. (2000). Baryogenesis. NATO Science Series, C555, 397-416.

Simonov, K., Capolupo, A., \& Giampaolo, S. M. (2019). Gravity, entanglement and CPT-symmetry violation in particle mixing. The European Physical Journal , C79, 902.

Steigman, G. (1976). Observational tests for antimatter cosmologies. Annual Review of Astronomy and Astrophysics 14, 339-372.

Steigman, G. (2007). Primordial nucleosynthesis in the precision cosmology era. Annual Review of Nuclear and Particle Science 57, 463-491.

Thomas, C., Dezen, T., Grohs, E. B., \& Kishimoto, C. T. (2020). Electron-positron annihilation freeze-out in the early universe. Physical Review D, 101, 063507.

Toussaint, D., Treiman, S. B., Wilczek, F., \& Zee, A. (1979). Matter-antimatter accounting, thermodynamics, and black hole radiation. Physical Review D, 19, 1036-1045.

Verlinde, E.P. (2011). On the origin of gravity and the laws of Newton, Journal of High Energy Physics, 04, 1104:029.

Wald, R. M. (1975). On particle creation by black holes. Communications in Mathematical Physics, 45, 9-45.

Weinberg, S. (1979). Cosmological production of baryons. Physical Review Letters 42, 850-853.

Weinberg, S. (1995, 1996, 2000). The quantum theory of fields I-III, Cambridge Univ. Press, Cambridge.

von Weizsäcker, C.F. (1939). Der zweite Hauptsatz und der Unterschied von Vergangenheit und Zukunft, Ann. Physik. 36, 275-283, reprinted in: Die Einheit der Natur, pp. 172-182, Hanser, München, Wien (1971)

Zeldovich, Ya. .B. (1971). Stars and relativity. Chicago: University Chicago Press.

Publisher's Note Springer Nature remains neutral with regard to jurisdictional claims in published maps and institutional affiliations. 
Gábor Etesi is a Hungarian mathematical physicist graduated from the Eötvös Roland University Budapest, Hungary in 1995. Currently he is a reader (associate professor) of geometry at the Department of Geometry, Mathematical Institute of the Budapest University of Technology and Economics. His main research field is mathematical problems of classical and quantum Yang-Mills theory and general relativity. For further information visit his homepage http://www.math.bme.hu/ etesi 